\title{
25 Research Square \\ p53 Missense Mutation is Associated with Immune Cell PD-L1 Expression in Triple-negative Breast Cancer
}

\section{Bin Wang}

The First Affiliated Hospital of Shandong First Medical University

Kun Wang

The First Affiliated Hospital of Shandong First Medical University

Jian Yu

The First Affiliated Hospital of Shandong First Medical University

Long Liu

Shandong University Qilu Hospital

Ke Liang

Shandong University Qilu Hospital

Ai-yan Xing ( $\square$ aiyan_1986@163.com )

Shandong University Qilu Hospital

\section{Research Article}

Keywords: p53 missense mutation, immune cell PD-L1 expression, triple-negative breast cancer

Posted Date: November 18th, 2021

DOI: https://doi.org/10.21203/rs.3.rs-1070659/v1

License: (c) (1) This work is licensed under a Creative Commons Attribution 4.0 International License.

Read Full License 


\section{Abstract}

\section{Background}

Immunotherapy is an important treatment for triple-negative breast cancer (TNBC). The programmed death ligand 1 (PD-L1) is a pivotal biomarker in TNBC, and its expression is closely related to immunotherapy response. Therefore, the association of p53 expression and mutation and PD-L1 expression was explored.

Methods and Results

PD-L1 expression and p53 mutation and expression were evaluated by immunohistochemistry. PD-L1 expression and expression levels between p53 mutation (missense and nonsense) and wild type; noexpression/loss vs. expression; and missense vs. nonsense groups were compared. PD-L1 expression was found mainly in tumor-infiltrating immune cells (ICs) in TNBC. Positive PD-L1 expression was associated with a high Ki67 index. There was a significant association between p53 mutation, especially missense mutation and higher histological grade, and PD-L1 expression in ICs. Compared with p53 nonsense mutation, cases with missense mutations tended to display more PD-L1 positive ICs. However, PD-L1 expression in ICs was not significantly different between the p53 mutation and expression groups.

Conclusions

p53 missense mutation is partially involved in the regulatory expression of PD-L1. Both p53 missense mutation and PD-L1 expression may be potential targets for improving immunotherapy response in TNBC.

\section{Introduction}

Triple negative breast cancer (TNBC) refers to breast cancer with negative immunohistochemical staining results for the estrogen receptor (ER), progesterone receptor (PR), and human epidermal growth factor receptor-2 (HER-2). TNBC accounts for 15-20\% of all pathological types of breast cancer [1]. At present, there is no specific targeted drug for TNBC, and clinical treatment is reliant on chemotherapy. Although some patients with TNBC show a partial pathologic complete response after neoadjuvant chemotherapy, the 5-year survival rate of TNBC patients is less than 30\% [2] when spread and metastasis occur.

Immunotherapy has played an increasingly important role in the clinical management of TNBC [3]. The programmed cell death protein 1 (PD-1)/programmed death ligand 1 (PD-L1) axis has been confirmed as a significant immune checkpoint, and high PD-L1 expression in tumor cells contributes to immune escape and poor prognosis. Therefore, patients with PD-1 expression or high PD-L1 expression may benefit from anti-PD-1/PD-L1 inhibitor treatment [4]. The detailed regulatory mechanism of PD-L1 expression in TNBC is currently unclear, and the factors closely related to the expression of PD-L1 are worth exploring. 
TP53 is a tumor suppressor gene that participates in the regulation of cell proliferation, the cell cycle, and DNA damage repair [5]. TP53 mutation is closely associated with tumorigenesis and progression. In melanoma, a higher frequency of PD-L1 positivity was observed in p53 mutated cells [6]. In oral squamous cell carcinoma, the expression of p53 and PD-L1 in tumor cells was significantly and positively correlated [7]. TP53 is reportedly a more commonly mutated gene; the p53 mutation rate is over $80 \%$ in TNBC [8]. Whether $p 53$ expression or mutation is associated with PD-L1 expression in TNBC requires verification.

In this study, we investigated the association between PD-L1 protein expression and p53 mutation (loss/nonsense and missense) or expression in TNBC. We also explored theoretical evidence for the function of p53 mutation in Chinese patients with TNBC undergoing immunotherapy.

\section{Methods}

\section{Patients and tissue specimens}

Samples were collected from 172 TNBC patients who underwent radical surgery between 2017 January and 2020 April at the Shandong University Qilu Hospital. Of the 172 cases, 17 patients $(9.88 \%)$ received neoadjuvant chemotherapy before surgery. Miller and Payne (MP) response assessment of these 17 cases was also evaluated, and the results showed that four cases were grade 1 , six cases were grade 2 , six cases were grade 3 , and one case was grade 4 . Clinicopathological characteristics of all samples were available from pathology reports and medical records (See Table 1). Informed consent was obtained, and the study was approved by The Ethics Committee of Shandong University. The procedures involving human subjects were in accordance with the Declaration of Helsinki.

\section{Immunohistochemistry}

Sections $(5 \mu \mathrm{m})$ from formalin-fixed paraffin-embedded blocks of 172 cases were used to measure the expression of ER, PR, HER-2, Ki67, p53, and PD-L1. Antibodies against ER (SP1, Ventana), PR (1E2, Ventana), HER-2/neu (4B5, Ventana), Ki67 (30-9, Ventana), P53 (D0-7, ZSGB-BIO), and PD-L1 (SP142, Abcam) were used for immunohistochemical evaluation. The staining was carried out on an automatic immunohistochemical staining device (Benchmark XT, Ventana Medical Systems, USA) according to standard procedures.

\section{Immunostaining evaluation}

ER and PR expressions were defined as negative if no cells or $<1 \%$ tumor cells had nuclear immunostaining. The immunostaining of HER2 was defined as: $0,1+$ immunostaining, and 2+ immunostaining but FISH negative. Specifically, 0 : No staining or $\leq 10 \%$ of infiltrating cancer cells present showed incomplete and weak cell membrane staining; $1+:>10 \%$ of infiltrating cancer cells 
present showed incomplete and weak cell membrane staining; $2+:$ > $10 \%$ of infiltrating cancer cells showed incomplete and/or weak to moderate cell membrane staining; or $\leq 10 \%$ of infiltrating cancer cells showed strong and complete cell membrane staining. Greater than or equal to $1 \%$ stained tumor cells (TCs) or ICs were defined as PD-L1 positive, whereas $<1 \%$ stained TCs or ICs were defined as PD-L1 negative [9]. The pattern of p53 expression in TNBC included mutated and wild types. Mutation showed two patterns: nonsense (loss) and missense mutation. Tumor cell nuclei stained as variable and weak intensity (regardless of stained cell proportion) were defined as the wild-type pattern. Negative staining of tumor cell nuclei was defined as nonsense (loss) mutation. Missense mutation was defined as $\geq 60 \%$ of tumor cell nuclei stained uniformly strong or with moderate intensity [10]. All immunostaining assessments were determined by two pathologists (P Gao and AY Xing).

\section{Statistical analysis}

Prism 5 (GraphPad Software, La Jolla, CA, USA) was used to perform statistical analyses. The expression of PD-L1 and p53 expression or mutation and pathological parameters were analyzed using the Chisquared test or Fisher's exact test. All tests were two-sided, and statistical significance was set at $P<$ 0.05 .

\section{Results}

\section{PD-L1 expression in TNBC}

Immunohistochemistry was performed to evaluate PD-L1 expression in 172 cases of TNBC. PD-L1 expression was observed in TCs and ICs. However, PD-L1 staining was mainly observed in the ICs of TNBC. Negative PD-L1 expression was found in 117 cases (68.02\%, Fig 1A), and positive PD-L1 expression was found in 55 cases $(31.98 \%$ ), including three cases with both positive PD-L1 expression of TCs and ICs (5.45\%; Fig 1C) and 52 cases with ICs (94.55\%, Fig 1B). No significant association was observed between PD-L1 expression and age, tumor size, lymph node metastasis, and histological grade (Table 1). However, there was an obvious association between PD-L1 expression and high Ki67 index ( $P<$ 0.05). The cases with PD-L1 expression had a higher Ki67 proliferation index, which suggested that these patients might have a poor prognosis.

\section{p53 mutation and expression in TNBC}

Of the 172 cases, 59 cases (34.30\%) expressed the p53 wild-type and 113 cases (65.70\%) expressed the p53 mutation-type, including 70 cases $(40.70 \%)$ with nonsense mutation and 43 cases $(25 \%)$ with missense mutation. Regarding expression, no-expression of p53 was found in 70 cases (40.70\%) and p53 expression was found in 102 cases (59.30\%). No significant difference was observed in age, tumor size, lymph node metastasis, and Ki67 index between the p53 mutation and wild-type groups $(P>0.05$; Table 2). However, there was a significant difference in histological grade between the p53 mutation and wild- 
type groups $(P<0.05$; Table 2$)$, which suggested that p53 mutation might be related to higher histological grade and poor prognosis. Further, we divided the mutation subtypes into two groups: nonsense and missense mutation. We found that there was a statistically significant difference in histological grade between the $\mathrm{p} 53$ nonsense and missense mutation groups $(P<0.05$; Table 2$)$. The cases with p53 missense mutations had a higher histological grade, indicating that p53 missense mutation might play an important role in tumorigenesis and development in TNBC. In addition, there was no significant difference observed in age, tumor size, lymph node metastasis, histological grade, and Ki67 index between the 553 no-expression and expression groups $(P>0.05$; Table 2$)$.

\section{PD-L1 expression of ICs is associated with p53 mutation}

To confirm whether PD-L1 expression is associated with p53 mutation or expression, a comparative analysis was performed. Of the 117 cases with PD-L1 negative expression, there were 23 cases (19.66\%) with p53 missense mutation, 52 cases $(44.44 \%)$ with p53 nonsense mutation, and 42 cases $(35.90 \%)$ with wild-type p53. Additionally, there were 20 cases (36.36\%) with p53 missense mutation, 18 cases (32.73\%) with p53 nonsense mutation, and 17 cases (30.91\%) with wild-type p53 in the PD-L1 positive expression group (55 cases total). In summary, p53 mutation was identified in 75 cases $(64.10 \%)$ in the PD-L1 negative group and 38 cases $(69.09 \%)$ in the PD-L1 positive group, and p53 expression was identified in 65 cases (55.56\%) in the PD-L1 negative group and 37 cases (67.27\%) in PD-L1 positive group. PD-L1 expression was not statistically different between the p53 mutation and wild-type groups, as well as the p53 no-expression and expression groups. However, a statistically significant difference in PD-L1 expression was found between the p53 missense and nonsense groups $(P<0.05$; Table 3), demonstrating that PD-L1 positive expression might be closely related to $\mathrm{p} 53$ missense mutation.

\section{PD-L1 expression level of ICs is not related to p53 mutation}

As mentioned above, IC PD-L1 expression level was classified into three groups: IC1, IC2, and IC3. The PDL1 expression level of ICs in the PD-L1 positive expression group was analyzed. Of the 55 cases, there were 27 cases (49.09\%) in the IC1 group (Fig 2A), 15 cases in the IC2 (27.27\%, Fig 2B) group, and 13 cases $(23.64 \%$, Fig 2 C) in the IC3 group. In the IC1 group, 10 cases $(37.04 \%)$ had p53 missense mutations, 9 cases $(33.33 \%)$ had p53 nonsense mutations, and 8 cases $(29.63 \%)$ were p53 wild type. In the IC2 group, six cases (40\%) had p53 missense mutations, four cases $(26.67 \%)$ had p53 nonsense mutations, and five cases (33.33\%) were p53 wild type. In the IC3 group, four cases (30.77\%) had p53 missense mutations, five cases $(38.46 \%)$ had p53 nonsense mutations, and four cases $(30.77 \%)$ were p53 wild type. In summary, 19 cases $(70.37 \%)$ had p53 mutations and 8 cases $(29.63 \%)$ were p53 wild type; 9 cases (33.33\%) had no expression of p53 and 18 cases (66.67\%) expressed p53 in the IC1 group. In the IC2 group, 10 cases (66.67\%) had p53 mutations and 5 cases (33.33\%) were p53 wild type; four cases $(26.67 \%)$ had no expression of $p 53$, while 11 cases $(73.33 \%)$ expressed $p 53$. In the IC3 group, nine cases $(69.23 \%)$ had p53 mutations and four cases (30.77\%) were p53 wild type; five cases (38.46\%) had 
no expression of p53, while eight cases (61.54\%) expressed p53 (Table 3). No significant difference was observed between the IC1, IC2, and IC3 groups, regardless of p53 mutation or expression status $(P>$ 0.05).

\section{Discussion}

Immunotherapy for TNBC has gained increasing attention from researchers in recent years. Based on gene expression profiles, studies have identified 'immunomodulatory' (IM) as a molecular subtype [11] of TNBC. Because the immune cell signaling pathways in this subtype are in an active state, these patients may benefit from immunotherapy and have a better prognosis compared to the basal-like immunosuppressive (BLIS) subtype [12]. PD-L1 is an important biomarker, and its expression level is closely associated with the response to immunotherapy. The determination of PD-L1 expression and the factors affecting its expression has important clinical value regarding immunotherapy.

In this study, we found that PD-L1 was mainly expressed in ICs but not in TCs in TNBC. Of the 55 cases with PD-L1 positive expression, only three cases (5.45\%) exhibited positive PD-L1 expression in the TCs. Moreover, positive PD-L1 expression in ICs was also observed in these three cases. Therefore, ICs and the microenvironment of TNBC require further study. Studies have shown that more tumor infiltrating lymphocytes (Tils) are found in or around the tumor stroma of the IM type of TNBC [13], and these lymphocytes are positive for CD8, indicating that they are highly immunogenic. Thus, PD-L1 positive expression and evaluation of ICs in TNBC might be clinically significant. The cases with PD-L1 positive expression displayed a higher Ki67 proliferation index, also suggesting more aggressive biological behavior.

TP53 is an important suppressor gene that plays a key role in physiological processes. When the TP53 gene is mutated, it loses its regulatory effect on cell growth, apoptosis, and DNA damage repair due to its spatial conformation changes, which may lead to tumorigenesis [14]. Thus, p53 mutants have become a new target for cancer therapies. Synnott et al. found that COTI-2, a third-generation thiosemicarbazone, acts by reactivating mutant p53 to its wild form and inhibits tumor cell growth in TNBC [15]. Notably, there are two p53 mutation types: nonsense and missense. Therefore, we sought to determine which form of p53 mutation is more important to clinical treatment. In this study, we collected data on the expression and mutation of $\mathrm{p} 53$ to further analyze its expression or mutation with clinicopathological variables and PD-L1 expression. We found that p53 mutation was associated with high histological grade compared with the p53 wild type. In particular, the cases with p53 missense mutations tended to have a higher histological grade. However, there was no significant association between p53 expression (no/loss and expression) and clinicopathological variables including age, tumor size, lymph metastasis, histological grade, and Ki67index. Thus, regardless of the p53 mutation type (missense or nonsense), mutation may be closely related to prognosis in TNBC.

Further, PD-L1 expression and p53 mutation or expression (mutation vs. wild; missense vs. nonsense; and no expression vs. expression) were compared and analyzed. The results showed no statistically 
significant difference in PD-L1 expression between the p53 mutation and wild-type groups, as well as the p53 no-expression and expression groups. However, between the p53 missense and nonsense groups, there was a significant difference in PD-L1 expression. Therefore, we have reason to believe that p53 mutation subtypes are critical to PD-L1 expression. A p53 missense mutation may lead to overexpression of PD-L1 in ICs. There was no significant difference in PD-L1 expression levels in ICs (IC1, IC2, and IC3) between p53 mutation and wild type, missense and nonsense, or the no-expression and expression groups. Recently, the association between p53 mutation and the number of Tils in TNBC was reported [16]. Miseon et al. [17] proposed a hypothesis that p53 mutation produces a mutated p53 protein, which acts as a neoantigen, potentially triggering the immune response and increasing Til levels. Studies with larger sample sizes and focused on molecular mechanisms are required to validate this theory.

\section{Conclusions}

The results of this study showed that p53 missense mutation, but not nonsense or expression, was related to PD-L1 positive expression of ICs in TNBC. These results suggest a role for p53 missense mutation in the regulatory expression of PD-L1. Both p53 missense and PD-L1 expression could be potential predictors for immunotherapy response in TNBC.

\section{Declarations}

\section{Authors' contributions}

AYX designed the study. KL and LL performed the experiment. BW, KW and JY collected and analyzed the data; BW wrote the manuscript, and AYX reviewed and edited the manuscript.

\section{Funding}

This work was supported by the Natural Science Foundation of Shandong Province (No. ZR2019PH033) and the Natural Science Foundation of China (No. 91902884).

\section{Data availability}

The data are available from the corresponding author upon reasonable contact.

\section{Conflict of interest}

The authors have no conflict of interest to declare. 


\section{Informed consent}

Informed consent was obtained, and the study was approved by The Ethics Committee of Shandong University.

\section{References}

1. Morris GJ, Naidu S, Topham AK et al (2007) Differences in breast carcinoma characteristics in newly diagnosed African-American and Caucasian patients: a single-institution compilation compared with the National Cancer Institute's Surveillance, Epidemiology, and End Results database. Cancer 110(4):876-884

2. Dent R, Trudeau M, Pritchard KI et al (2007) Triple-negative breast cancer: clinical features and patterns of recurrence. Clin Cancer Res 13(15 pt 1):4429-4434

3. Keenan TE, Tolaney SM (2020) Role of Immunotherapy in Triple-Negative Breast Cancer. J Natl Compr Canc Netw 18(4):479-489

4. Mittendorf EA, Philips AV, Meric-Bernstam F et al (2014) PD-L1 expression in triple-negative breast cancer. Cancer Immunol Res 2(4):361-370

5. Pan Y, Yuan Y, Liu G, Wei Y (2017) P53 and Ki-67 as prognostic markers in triple-negative breast cancer patients. PLoS ONE 12(2):e0172324

6. Thiem A, Hesbacher S, Kneitz H et al (2019) IFN-gamma-induced PD-L1 expression in melanoma depends on p53 expression. J Exp Clin Cancer Res 38(1):397

7. Tojyo I, Shintani Y, Nakanishi T et al (2019) PD-L1 expression correlated with p53 expression in oral squamous cell carcinoma. Maxillofac Plast Reconstr Surg 41(1):56

8. Xiao G, Lundine D, Annor GK et al (2020) Gain-of-Function Mutant p53 R273H Interacts with Replicating DNA and PARP1 in Breast Cancer. Cancer Res 80(3):394-405

9. Narayan P, Wahby S, Gao JJ et al (2020) FDA approval summary: atezolizumab plus paclitaxel protein-bound for the treatment of patients with advanced or metastatic TNBC whose tumors express PD-L1. Clin Cancer Res 26:2284-2289

10. Darb-Esfahani S, Denkert C, Stenzinger A et al (2016) Role of TP53 mutations in triple negative and HER2-positive breast cancer treated with neoadjuvant anthracycline/taxane-based chemotherapy. Oncotarget 7:67686-67698

11. Lehmann BD, Jovanović B, Chen X et al (2016) Refinement of Triple-Negative Breast Cancer Molecular Subtypes: Implications for Neoadjuvant Chemotherapy SelectionJ. PLoS ONE 11(6):e0157368

12. Burstein MD, Tsimelzon A, Poage GM et al (2015) Comprehensive genomic analysis identifies novel subtypes and targets of triple-negative breast cancer. Clin Cancer Res 21(7):1688-1698

13. O'Meara T, Safonov A, Casadevall D et al (2019) Immune microenvironment of triple-negative breast cancer in African-American and Caucasian women. Breast Cancer Res Treat 175(1):247-259 
14. Oros Klein K, Oualkacha K, Lafond MH et al (2016) Gene Coexpression Analyses Differentiate Networks Associated with Diverse Cancers Harboring TP53 Missense or Null Mutations. Front Genet 7:137

15. Synnott NC, O'Connell D, Crown J, Duffy MJ (2020) COTI-2 reactivates mutant p53 and inhibits growth of triple-negative breast cancer cells. Breast Cancer Res Treat 179(1):47-56

16. Shah CA, Allison KH, Garcia RL et al (2008) Intratumoral T cells, tumor-associated macrophages, and regulatory T cells: association with p53 mutations, circulating tumor DNA and survival in women with ovarian cancer. Gynecol Oncol 109(2):215-219

17. Miseon Lee IA, Park S-H, Heo et al (2019) Association between p53 Expression and Amount of Tumor-Infiltrating Lymphocytes in Triple-Negative Breast Cancer. J Pathol Transl Med Actions Search in PubMed Search in NLM Catalog Add to Search 53(3):180-187. doi: 10.4132/jptm.2019.02.08

\section{Tables}

Due to technical limitations, tables are only available as a download in the Supplemental Files section.

\section{Figures}
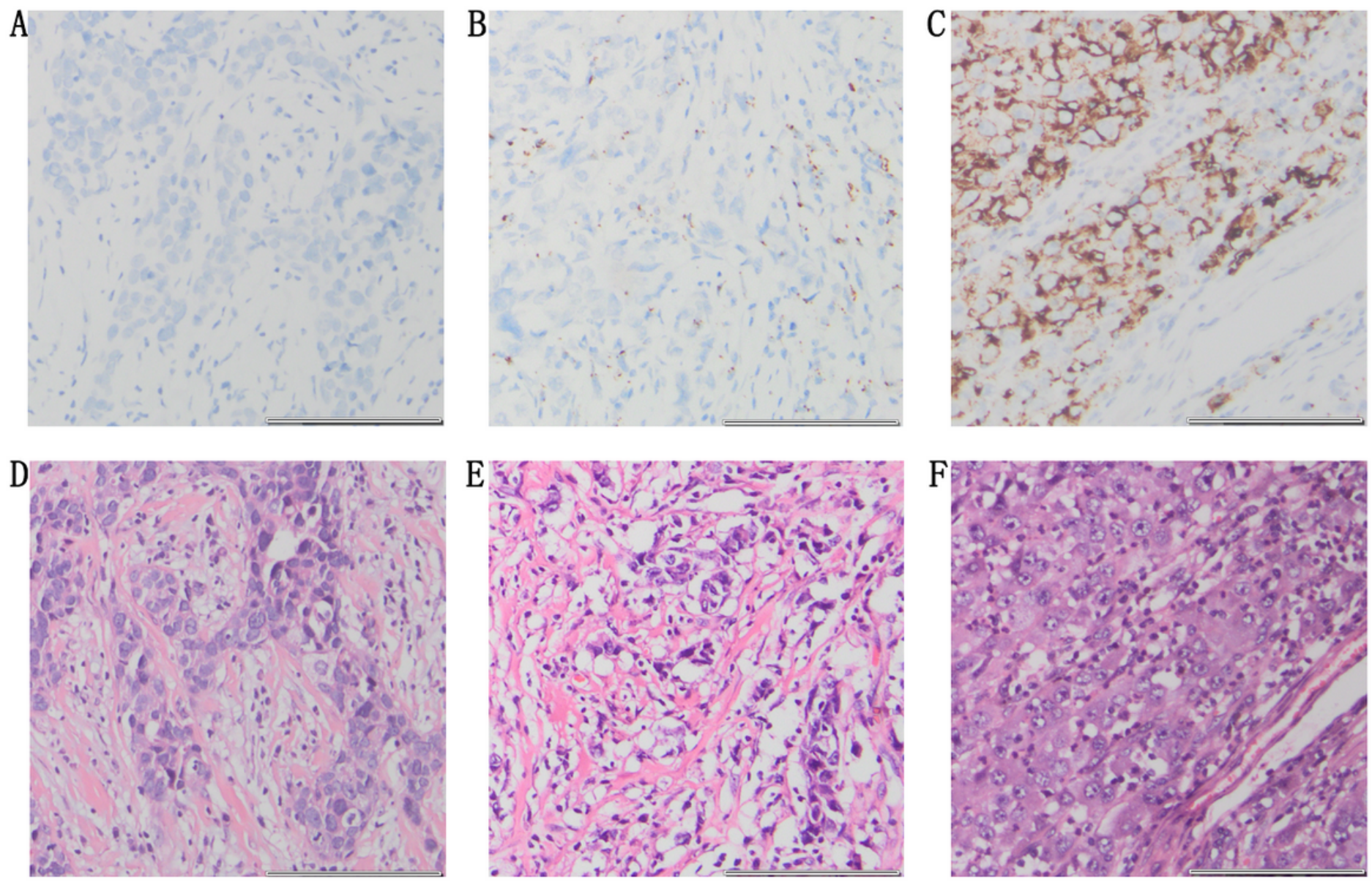


\section{Figure 1}

PD-L1 expression in TNBC. Negative expression of PD-L1 in both TCs and ICs (A). Negative expression of PD-L1 in TCs, but positive expression of PD-L1 in ICs (B). Positive expression of PD-L1 in both TCs and ICs (C). Corresponding eosin-hematoxylin staining of the three cases is shown (D, E, F). The scale bar represents 500 pixels.
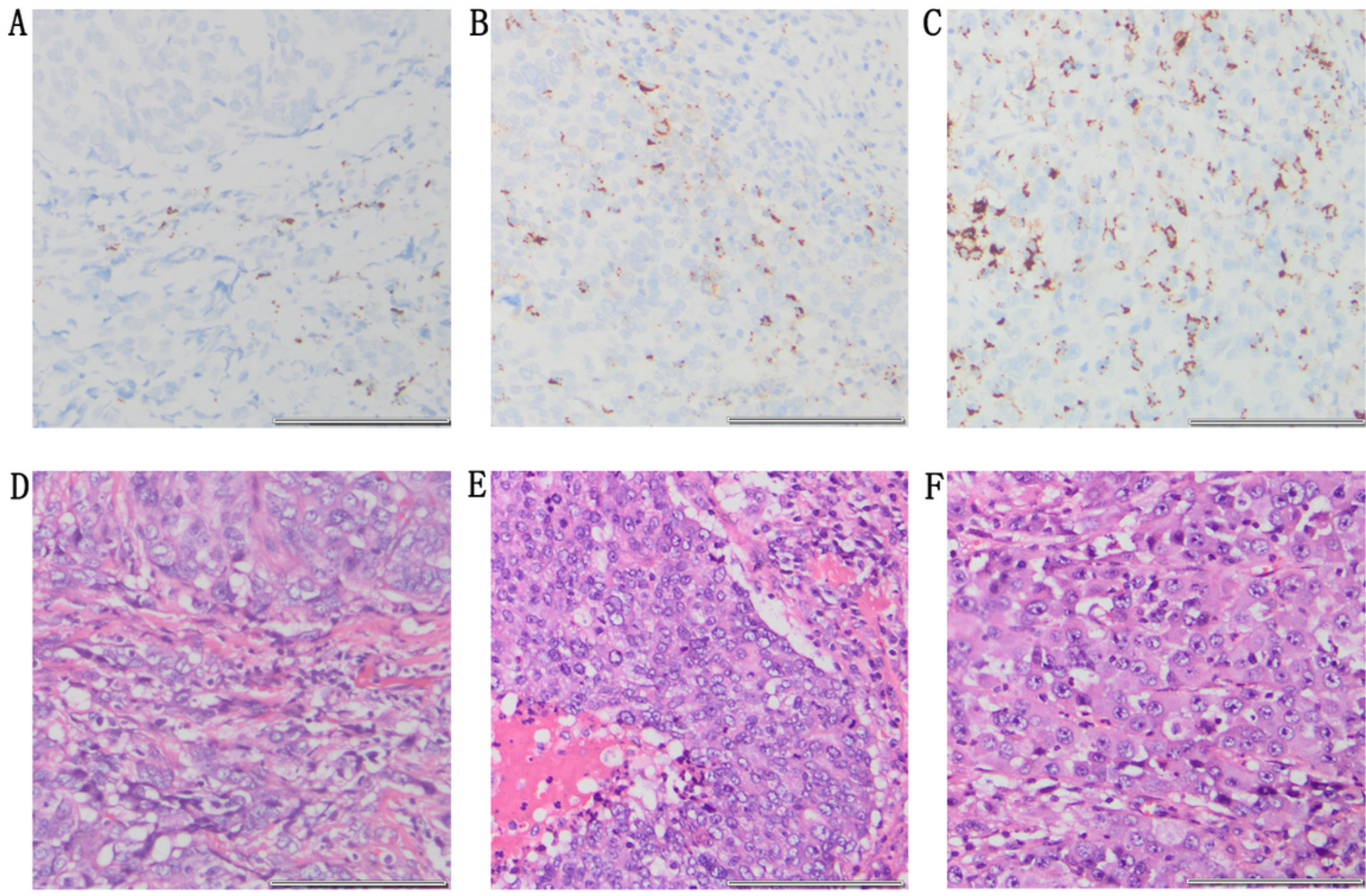

Figure 2

The expression levels of PD-L1 in ICs. One percent positive staining of PD-L1 in ICs (IC1, A), 5\% positive staining of PD-L1 in ICs (IC2, B), and 10\% positive staining of PD-L1 in ICs (IC3, C). Corresponding eosinhematoxylin staining of the three cases is shown (D, E, F). The scale bar represents 500 pixels.

\section{Supplementary Files}

This is a list of supplementary files associated with this preprint. Click to download.

- Table1.docx

- Table2.docx

- Table3.docx 